\title{
Neutrino CP Violation with the European Spallation Source neutrino Super Beam project
}

\author{
Marcos Dracos*广 \\ IPHC, Université de Strasbourg, CNRS/IN2P3, F-67037 Strasbourg, France \\ E-mail: marcos.dracosein2p3.fr
}

\begin{abstract}
After measuring in 2012 a relatively large value of the neutrino mixing angle $\theta_{13}$, the door is now open to observe for the first time a possible $\mathrm{CP}$ violation in the leptonic sector. The measured value of $\theta_{13}$ privileges the 2nd oscillation maximum for the discovery of CP violation instead of the usually used 1 st oscillation maximum. The sensitivity at this 2 nd oscillation maximum is about three times higher than for the 1st oscillation maximum inducing a lower sensitivity to systematic errors. Going to the 2 nd oscillation maximum necessitates a very intense neutrino beam with the appropriate energy. The world's most intense pulsed spallation neutron facility, the European Spallation Source, will have a proton linac with $5 \mathrm{MW}$ power and $2 \mathrm{GeV}$ energy. This linac, under construction, also has the potential to become the proton driver of the world's most intense neutrino beam with very high probability to discover a neutrino $\mathrm{CP}$ violation. The physics performance of this neutrino Super Beam in conjunction with a megaton underground Water Cherenkov detector installed at a distance of about $500 \mathrm{~km}$ from ESS, has been evaluated. In addition, the choice of such detector will extend the physics program to proton-decay, atmospheric neutrinos and astrophysics searches. The ESS proton linac upgrades, the accumulator ring needed for proton pulse compression, the target station and the physics potential are described. In addition to neutrinos, this facility will also produce at the same time a copious number of muons which could be used by a muon collider. The ESS neutron facility will be fully ready by 2025 at which moment the upgrades for the neutrino facility could start.
\end{abstract}

XXIX International Symposium on Lepton Photon Interactions at High Energies - LeptonPhoton2019 August 5-10, 2019

Toronto, Canada

\footnotetext{
* Speaker.

$\dagger$ This project is supported by the COST Action EuroNuNet "Combining forces for a novel European facility for neutrino-antineutrino symmetry-violation discovery". It has also received funding from the European Union's Horizon 2020 research and innovation programme under grant agreement No 777419 .
} 


\section{Introduction}

The last measured neutrino mixing parameter $\theta_{13}$ has been found to be relatively high compared to expectations. In this landscape the sensitivity to $\mathrm{CP}$ violation observation and measurement of the violating parameter $\delta_{C P}$, is enhanced at the second oscillation maximum compared to observations at the first one $[1,2,3]$. Moreover, by placing the far detector at the second oscillation maximum, the experiment is significantly less affected by systematic uncertainties. This is an important point since improvement of the present systematic errors is known to be very hard.

The drawback by placing the far detector at the 2 nd oscillation maximum comes from the fact that very high intensity neutrino beams are needed to compensate for the longer baseline. The European Spallation Source (ESS) neutron facility under construction in Lund, Sweden, will have a $5 \mathrm{MW}, 2 \mathrm{GeV}$ proton linac operated at a rate of $14 \mathrm{~Hz}$ (4\% duty cycle). This linac could also be used to produce an intense neutrino beam, which, combined with a megaton Water Cherenkov detector placed at a distance of about $500 \mathrm{~km}$ (second oscillation maximum), could observe for the first time CP violation in the leptonic sector. This could help to understand the matter-antimatter asymmetry in the Universe. This project, called ESS neutrino Super Beam (ESSvSB) [4], has been proposed after measuring the last mixing angle $\theta_{13}$ and it is under optimisation for an exclusive operation on the 2 nd oscillation maximum.

\section{The $\operatorname{ESS} v \mathrm{SB}$ project}

Taking into account the low duty cycle of the ESS proton linac, the ESS $v$ SB project proposes to double the proton pulse frequency from $14 \mathrm{~Hz}$ to $28 \mathrm{~Hz}$. In this way, the facility will send one pulse for neutron production and one pulse to the neutrino facility. To produce a neutrino beam, the ESS proton linac needs some modifications on top of the cooling power upgrades. Due to limitations at the level of the hadron collector of the neutrino facility, the proton bunches have to be compressed from $2.86 \mathrm{~ms}$, used for neutron production, to few $\mu \mathrm{s}$ for the neutrino beam. For this, an accumulation ring is needed to be placed after the linac and before the neutrino target station. The presence of an accumulator obliges to use $\mathrm{H}^{-}$ions instead of protons to avoid space charge effects during the injection in the accumulator.

An evaluation of all required upgrades of the linac can be found in a CERN note [7]. This report also makes the recommendation to increase the proton energy from $2 \mathrm{GeV}$ to $2.5 \mathrm{GeV}$ in order to reduce space charge effects. This proton energy is now considered as the baseline of the $\mathrm{ESS} v \mathrm{SB}$ project.

A neutrino target station including a hadron decay tunnel has to be placed just downstream of the accumulation ring. The adopted design is the one proposed by the EU Design Study EUROv [5]. It consists of four targets and magnetic horns hit alternatively by the proton pulses. The four target/horn scheme has been adopted in order to mitigate the effect of the $5 \mathrm{MW}$ proton beam. The length of the decay tunnel, $25 \mathrm{~m}$, has been optimised to increase the number of decaying pions, producing muon neutrinos, and keeping the number of decaying muons, producing electron neutrinos, to a limited level.

The MEMPHYS type detector (Water Cherenkov) has been adopted as far detector in order to evaluate the physics performance of the proposed facility. The neutrino detection performance 
of this detector can be found in [6]. Compared to this performance evaluated several years ago, the MEMPHYS detection capability could now be significantly improved for the same cost, by increasing the number of photomultipliers with furthermore higher Quantum Efficiency, profiting from recent developments on this subject. This will significantly improve the electron neutrino detection efficiency and thus increase the physics performance of the facility.

The neutrino beam is directed towards the north of Sweden in the direction of the Garpenberg mine, $540 \mathrm{~km}$ away, which could host the far detector. Another alternative location is the Zinkgruvan mine at $360 \mathrm{~km}$ from Lund.

\section{Physics Performance}

The neutrino oscillation to be studied here is $v_{\mu} \rightarrow v_{e}$. Fig. 1 presents the unoscillated neutrino energy distribution which could be obtained by the proposed facility at an arbitrary on-axis distance of $100 \mathrm{~km}$ from the neutrino target. This distribution corresponds to one year neutrino run (200 days). An almost pure muon neutrino beam is produced with a main contamination of about $0.5 \%$ of electron neutrinos. This contribution polluting the primary muon neutrino beam, could be used to measure the electron neutrino cross-section using a perfomant near detector.


Figure 1: Neutrino energy distribution for neutrino (left) and anti-neutrino (right) runs at an arbitrary distance of $100 \mathrm{~km}$ from the target station, for $2.5 \mathrm{GeV}$ protons.

Fig. 2 presents the $v_{\mu} \rightarrow v_{e}$ oscillation probability at a distance of $540 \mathrm{~km}$ for several values of $\delta_{C P}$ and for normal and inverted neutrino mass hierarchies. The overlapping grey distribution is the $v_{e}$ energy distribution coming from the $v_{\mu}$ oscillation. It is well seen that the 2 nd oscillation maximum is fully covered. From this figure it is also seen that the $\mathrm{CP}$ violation discovery potentiality is not affected by the unknown neutrino mass hierarchy. It has to be mentioned that this project is exclusively devoted to $\mathrm{CP}$ violation discovery and not to the masse hierarchy determination which is believed to be solved by then by experiments supposed to start taking data during the next decade.

The physics performance of all projects strongly depends on the considered systematic uncertainties. As said before, systematic uncertainties play less a role on the 2nd oscillation maximum, thanks to the interference term in the oscillation probability dominating the solar and atmospheric 


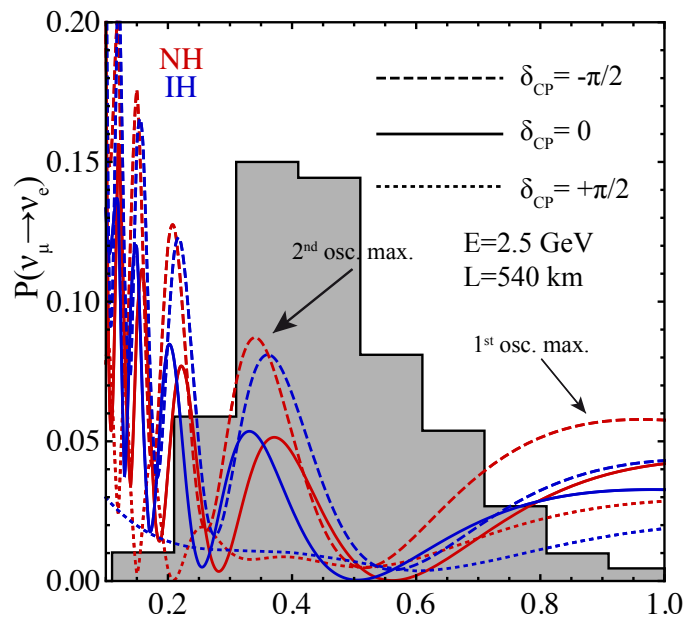

Figure 2: $\quad v_{\mu} \rightarrow v_{e}$ oscillation probability as a function of the neutrino energy. The red (blue) lines are for normal hierarchy (inverted). The shaded histogram is the energy distribution of $v_{e}$ produced by the $v_{\mu}$ oscillation and detected by the far detector.



Figure 3: The significance with which $\mathrm{CP}$ violation can be discovered as function $\delta_{C P}$.

terms [2]. For this evaluation the systematic errors reported in publication [8] have been considered, with mainly $5 \%$ error on the signal. After 10-years operation, it is expected that about 600 electron neutrinos and antineutrinos will be detected by the far detector. Fig. 3 slows the CP violation discovery significance versus $\delta_{C P}$. Up to $60 \%$ of the $\delta_{C P}$ values can be covered with a significance of $5 \sigma$. Studies are under way to increase the number of detected neutrinos by further optimising mainly the magnetic horn shape and the far detector performance.

\section{References}

[1] H. Nunokawa, S. J. Parke and J. W. F. Valle, "CP Violation and Neutrino Oscillations," Prog. Part. Nucl. Phys. 60 (2008) 338 doi:10.1016/j.ppnp.2007.10.001.

[2] P. Coloma and E. Fernandez-Martinez, "Optimization of neutrino oscillation facilities for large $\theta_{13}$," JHEP 1204 (2012) 089 doi:10.1007/JHEP04(2012)089.

[3] S. Parke, "Neutrinos: Theory and Phenomenology", Phys. Scripta T 158 (2013) 014013 doi:10.1088/0031-8949/2013/T158/014013 [arXiv:1310.5992 [hep-ph]].

[4] E. Baussan et al., "A Very Intense Neutrino Super Beam Experiment for Leptonic CP Violation Discovery based on the European Spallation Source Linac", Nucl. Phys. B885 (2014) 127-149.

[5] E. Baussan et al. [EUROnu Super Beam Collaboration], arXiv:1212.0732 [physics.acc-ph].

[6] L. Agostino et al., "Study of the performance of a large scale water-Cherenkov detector (MEMPHYS)", JCAP 1301, 024 (2013).

[7] F. Gerigk and E. Montesinos, "Required modifications of the ESS accelerator architecture for ESSnuSB", CERN-ACC-NOTE-2016-0050, July 2016.

[8] P. Coloma, P. Huber, J. Kopp and W. Winter, Phys. Rev. D 87 (2013) 3, 033004. 